\title{
Role of Topical Steroids, Anti Virals in Treating Acute Viral Conjunctivitis in Initial Presentation
}

\section{Bastola $\mathbf{P}^{1 *}$, Koirala $\mathbf{S}^{2}$, Lucky $\mathrm{KK}^{3}$ and Gupta $\mathrm{KK}^{4}$}

${ }^{1}$ Head of Department, Associate Professor, MD, MPH, DLSHTM, Consultant Ophthalmologist, Department of Ophthalmology, Nepalgunj Medical College and Teaching Hospital, Kohalpur, Banke, Nepal

${ }^{2}$ Associate Professor, MD, DM, National Academy of Medical Sciences, Bir Hospital, Kathmandu, Nepal

${ }^{3} \mathrm{MS}$, Ophthalmologist, Anterior Segment Fellow, Biratnagar Eye Hospital, Nepal

${ }^{4} \mathrm{MS}$, Ophthalmologist, Kedia Eye Hospital, Nepal

*Corresponding Author: Pradeep Bastola, Head of Department, Associate Professor, MD, MPH, DLSHTM, Consultant Ophthalmologist, Department of Ophthalmology, Nepalgunj Medical College and Teaching Hospital, Kohalpur, Banke, Nepal.

Received: June 22, 2019; Published: September 26, 2019

DOI: $10.31080 /$ ASOP.2019.02.0068

\begin{abstract}
Background: Acute viral conjunctivitis is the commonest infectious conjunctivitis. The commonest cause of epidemic acute viral conjunctivitis is adeno virus. Acute viral conjunctivitis cases are mostly self-limiting with conservative treatment; however, few cases possess significant challenge for proper management.

Aim/Objective: The study aimed to find out the response conventional versus topical steroids, antivirals and antibiotics therapy in managing acute cases of viral conjunctivitis.

Methods: This was a descriptive interventional study with intention to treat; the study period was from October 2014 to April 2016 , in the department of Ophthalmology, Nepalgunj Medical College and Teaching Hospital, Nepal. The participants enrolled in the study were blinded regarding treatment and were followed up up to five weeks. Intervention in arm one was conventional treatment, whereas intervention in arm two was steroid, antivirals topically besides placebo treatment.

Results: A total of 650 subjects (1300 eyes) were included in the study over a period of two and half years; gender wise 57.7\% (375) were males. Age group ranged from five years to 70 years. 193 (29.7\%) subjects presented with unilateral eye involvement. 90\% (585) study subjects had a positive history of contact with a subject with viral conjunctivitis. Redness (100\%), itchy eyes (69.2\%), ocular discomfort (53.8\%) were the main symptoms. There was a statistically significant difference in the two-treatment arm regarding the response of the treatment, $\mathrm{P}<0.05$.

Conclusion: The study could conclude that initial aggressive therapy using topical steroid combined with antibiotics and antivirals could not only prevent fellow eye getting acutely infected, but also prevents corneal involvement in subjects of acute viral conjunctivitis, regardless of the initial presentation of the eye involved, and the virus involved, and is a safe treatment option under supervision. Keywords: Acute Viral Conjunctivitis; Adeno Virus; Antibiotics Eye Drops; Keratitis; And Steroid Eye Drops
\end{abstract}

\section{Introduction}

Viral conjunctivitis is the commonest cause of infectious conjunctivitis both in overall and in the adult population. Approximately $65-90 \%$ of the cases of acute viral conjunctivitis are due to adeno viruses [1]. The condition is characterized by its highly contagious nature, redness of the eyes, serous discharge from the eyes, and history of family member affected which may be sometimes associated with mild grade fever, pain in front of the ears or pharyngitis. Importantly, there is no decrease in the vision of the affected ones in initial presentation [1-7]. Herpes simplex viruses comprises $1.3 \%$ to $4.8 \%$ of all cases of acute conjunctivitis $[5,6]$.
The differences in the transmissibility of bacterial and viral conjunctivitis merit careful consideration. Ocular adenoviral infection represents a significant public health problem in the US and worldwide. Although exact numbers are difficult to determine, estimations suggest the number of cases may be as high as $15-20$ million per year in the United States. Adenovirus conjunctivitis is a reportable infection in Germany and is classified as a Category IV infectious disease by Japan's National Epidemiological Surveillance of Infectious Diseases (NESID) with mandated collection, analysis and publication of reports on occurrences [8-16]. 
Several therapeutic agents have been evaluated for acute viral conjunctivitis; however, none to date have been approved for therapeutic use in humans $[9,10,17]$.

The frequency of viral conjunctivitis and its associated patient discomfort and lost days at work or school, a cure is constantly being searched for [1-7]. Unfortunately, the mainstay of therapy today is limited to ameliorating the symptoms until the natural course resolves. Many physicians found topical steroids to be helpful for patients with severe conjunctivitis. By reducing inflammation steroids can bring dramatic improvement in patient comfort, however study in rabbit models have shown that topical steroid might prolong ocular shedding of adenovirus by weeks. A placebo control randomized trial study in United Kingdom found that topical steroids were not harmful in treating presumed viral conjunctivitis cases [18-21]. The role of topical steroids along with topical antivirals for acute viral conjunctivitis at initial presentation is not harmful [21], infact it is positive [18,19], which formed the basis for this study.

The gold standard for treatment for acute viral conjunctivitis is to go for a symptomatic treatment as the disease is self-limiting without use of steroids topically at initial presentation. However, it has been observed in the last few years that the course of acute viral conjunctivitis has been more threatening than it has been in the past with many patients going in to develop corneal complications and eventually needing topical steroids along with topical anti-viral agents for the problems as once corneal complications sets in in the form of keratitis topical steroids and topical anti-viral are the main stay of treatment [17].

Keeping the backdrop in the mind, this study focused on treating these acute viral conjunctivitis cases with mild-moderate topical steroids, antivirals and ocular soothing agents at initial presentation only, the study primarily was undertaken with an intention to treat, in the treatment arm or arm two with a close monitoring of all the study subjects with regard to treatment response.

\section{Methods/Methodology}

The study was descriptive interventional study, with an intention to treat, which was carried out in the clinical setting of Ophthalmology outpatient department (OPD) of Nepalgunj Medical College and Teaching Hospital (NGMCTH) from October 2014 to April 2016 for a period of approximately two and half years. All the patients were clinically diagnosed to have acute viral conjunctivitis and were enrolled in the study. Patients below the age of five years and above 80 years of age were excluded from the study, other exclusion criteria were patients with history of treatment before presenting to the Ophthalmology outpatient department, patients with chronic debilitating disease or immunocompromised status, patients with concomitant bacterial/fungal conjunctivitis patients not willing to give an informed consent and patients losing follow up in the first follow up were excluded from the study as well.

The study firmly adhered to the tenets of Helsinki declaration following the ethics of a study. An informed consent was taken from all the study subjects, an internal approval was taken from the medical college to conduct the study ethically. The study subjects were told about the treatment protocol and were advised accordingly to follow up. It was a single blinded study where only the subjects did not know in which arm they were allocated.

A simple randomization was done to put study subjects in two arms, arm one (conventional treatment arm group), arm two (interventional treatment group). Computer-generated random numbers were used for randomization purpose. Arm one of the study subjects were treated with topical soothing agents, anti histaminics and mild topical antibiotic. Whereas, the other arm of subjects were treated with topical antivirals (3\% acyclovir) and topical dexamethasone and chloramphenicol drops $(0.1 \%+0.5 \%$ strengths) along with topical antihistaminic in the form of Naphazoline eye drops. In both the arms, the subjects were advised to be isolated, not to go to public places, and wear dark glasses or solar glasses to avoid contamination and transfer of disease to healthy eyes. During follow up periods, once the positive results of the intervention arm was established, the other arm subjects were also given the intervention arm treatment.

In all subjects both the eyes were treated irrespective of the initial presentation due to very high contagious nature of the disease in study.

All the subjects were advised to follow up at one-week time, three weeks' time minimum and the last follow up was advised only in patients who needed to change medications after three weeks. A total of three follow ups at one week, third week and fifth week were carried out.

Follow up closely focused on the patients response to hyperemia, perceived level of ocular discomfort, any serious complications using a questionnaire. A physician graded conjunctival hyperemia using a grading scale. 
Definitions/Protocols used in the study

Acute viral conjunctivitis:

Acute congestion of the eye (Unilateral or Bilateral) with serous discharge, clinically presence of chemosis, follicles (Inferior tarsus more), pseud-membrane, punctate epithelial erosions, hemorrhagic spots in the eye, with or without pharyngitis or local lymphadenopathy or lid swelling in the presence of normal visual acuity [11].

\section{Intervention Protocol and dosage}

Conventional Treatment (Control or First Arm)

Topical soothing agent (Antihistaminic) four times a day + Ciprofloxacin $(0.3 \%)$ or Chloramphenicol $(0.5 \%)$ four times a day + Non-steroidal anti-inflammatory drugs (NSAIDS) three times a day post food + Dark glasses to be used in day time only.

\section{Intention to Treat Arm (Second Arm)}

Dexamethasone $(0.1 \%)+$ Chloramphenicol $(0.5 \%)$ six to eight times a day, topical acyclovir (3\%) three times a day, antihistaminic four times a day, NSAIDs orally three times a day post food and dark glasses in day time.

In unilateral cases; both eyes were treated with same dosage of medications to prevent transmission of infection to fellow eye. In cases presenting with enlarged lymph nodes, pharyngitis and fever were treated symptomatically as well.

\section{Positive response to the treatment}

The subjects were given a questionnaire to respond about the treatment response in terms of ocular discomfort, conjunctival hyperemia, any serious complications with treatment, visual acuity, photophobia. The conjunctival hyperemia was graded by a physician. Looking at the response of treatment in both the arms that is conventional treatment arm (arm one) and interventional arm (arm two), a positive response was defined as where the patients ocular discomfort was reduced significantly, when the patients did not develop any further complications and the conjunctival hyperemia was reduced besides other symptoms and clinical signs.

Conjunctival Hyperemia and Conjunctival Chemosis Grading Score used in the Study

1. No Hyperemia, normal conjunctiva.

2. Mild Hyperemia without conjunctival chemosis.

3. Moderate Hyperemia with mild conjunctival chemosis.

4. Severe Hyperemia with moderate chemosis.

5. Very severe hyperemia with severe chemosis.

\section{Chemosis}

Edema of the bulbar conjunctiva.

\section{Follicles}

Lymphoid aggregates giving rise to a nodule more prominently seen in the lower tarsus.

\section{Pseudomembrane}

A superficial deposit of necrotic debri, inflammatory cells giving rise to swollen eye lids present in the upper or lower tarsus as a whitish membrane which can be peeled off under topical anesthetic eye drops easily without bleeding.

\section{Pharyngoconjunctival fever}

Conjunctivitis, pharyngitis and lymphadenopathy due to adeno viral infection [13].

The data was entered in a proforma designed for the study and later entered in SPSS version 22. Statistical analysis was done as per need; statician was consulted only when needed.

\section{Results}

A total of 650 subjects (1300 eyes) were enrolled in the study, all the 1300 eyes of the study subjects were treated. Gender wise 375 (57.7\%) were males followed by 275 (42.3\%) females. Age group ranged from five years to 70 years with mean being 27 years. Chief presenting complaints were redness in one eye or both in all subjects (100\%) followed by, itchy eyes 450 (69.2\%) discomfort in 350 (53.8\%), serious discharge 300 (46.1\%), lid swelling 250 (38.5\%), pain in front of the ear 115 (17.7\%), fever 95 (14.6\%), cough 73 (11.2\%), severe chemosis of the conjunctiva 65 (10\%), rapid progression 57 (87.7\%), pharyngeal symptoms 39 (6.0\%). Punctate epithelial lesions 25 (3.8\%), Unilateral ocular involvement was seen in 193 (29.7\%) whereas in rest subjects bilateral ocular involvement was seen (Table 1 ).

The subjects had different treatment protocol in two study arms and had varied response in one week and three weeks, looking at the response and scores (Table 2). Important finding in the study was there was no loss of follow up in all subjects enrolled.

\section{Discussion}

In the present study, male study subjects 375 (57.7\%) outnumbered the female study subjects slightly. The main presenting clinical features in the study were redness $100 \%$ study subjects followed by itchy eyes in $69.2 \%$, ocular discomfort in $53.8 \%$ and serous discharge in $46.1 \%$. All the study subjects when presented 


\begin{tabular}{|c|c|c|c|}
\hline \multicolumn{4}{|c|}{ Study Subjects $\dagger(N=650)$ in the current study and salient features } \\
\hline \multicolumn{4}{|c|}{ Total Number of Eyes Treated/Diagnosed $\neq(n=1300)$} \\
\hline Variables/Symptoms/Signs/Others & Present/Yes & Absent/No & Percentage (\%) \\
\hline Laterality: Unilateral (U), Bilateral (B) & \multicolumn{3}{|c|}{$193(29.7 \%$ U), 457 (70.3\% B) } \\
\hline Total Number of eyes treated & $1300(100 \%)$ & 0 & 100 \\
\hline Visual acuity impairment (Normal= N) & $0(0 \%)$ & $650(N, 100 \%)$ & \\
\hline Itchy eyes & $450(69.2 \%)$ & $200(30.8 \%)$ & 100 \\
\hline Redness (Subjects) & $650(100 \%)$ & 0 & 100 \\
\hline Ocular Discomfort & $350(53.8 \%)$ & $300(46.1 \%)$ & 100 \\
\hline Lid Swelling & $250(38.5 \%)$ & $400(61.5 \%)$ & 100 \\
\hline Serous discharge & $300(46.1 \%)$ & $350(53.8 \%))$ & 100 \\
\hline Pain in front of the ears & $115(17.7 \%)$ & $535(82.7 \%)$ & 100 \\
\hline Fever & $95(14.6 \%)$ & $555(85.3 \%)$ & 100 \\
\hline Cough & $73(11.2 \%)$ & $577(88.8 \%)$ & 100 \\
\hline Severe Chemosis & $65(10.0 \%)$ & $585(90.0 \%)$ & 100 \\
\hline Preauricular/Post auricular lymph node enlarged & $47(7.2 \%)$ & $603(92.8 \%)$ & 100 \\
\hline Conjunctival pseudo membrane & $45(6.9 \%)$ & $605(93.0 \%)$ & 100 \\
\hline Pharyngeal Symptoms & $39(6.0 \%)$ & $611(94.0 \%)$ & 100 \\
\hline Punctate epithelial lesions & $25(3.8 \%)$ & $625(96.2 \%)$ & 100 \\
\hline
\end{tabular}

Table 1: Showing salient features of the study subjects at first Presentation. $\dagger=$ Total number of study subjects in the current study. $\neq=$ Total number of the eyes treated in different arms in the study.

\begin{tabular}{|c|c|c|c|c|c|c|c|c|c|c|}
\hline \multirow[b]{2}{*}{ Variable } & \multirow[b]{2}{*}{ Category } & \multicolumn{3}{|c|}{ First week $(n=650)$} & \multicolumn{3}{|c|}{ Third week $(\mathrm{n}=650)$} & \multicolumn{3}{|c|}{ Fifth week $(\mathrm{n}=650)$} \\
\hline & & Arm-1 & Arm-2 & $P$ value & Arm-1 & Arm-2 & $P$ value & Arm-1 & Arm-2 & $P$ value \\
\hline \multirow{2}{*}{ Visual Acuity } & Normal & 325 & 325 & & 200 & 325 & \multirow{2}{*}{$<0.05$} & 200 & 450 & \multirow{2}{*}{$<0.05$} \\
\hline & Impaired & 0 & 0 & & $125^{*}$ & 0 & & 0 & 0 & \\
\hline \multirow[t]{5}{*}{$\begin{array}{l}\text { Congestion (Redness) } \\
\text { and chemosis of eye } \\
\text { involved }\end{array}$} & $\begin{array}{l}\text { No congestion and } \\
\text { chemosis }\end{array}$ & 35 & 125 & \multirow{5}{*}{$<0.05$} & 205 & 325 & \multirow{5}{*}{$<0.05$} & 205 & 445 & $<0.05$ \\
\hline & $\begin{array}{l}\text { Mild congestion and } \\
\text { chemosis }\end{array}$ & 40 & 150 & & $70^{*}$ & 0 & & 0 & 0 & \\
\hline & $\begin{array}{l}\text { Moderate congestion } \\
\text { and chemosis }\end{array}$ & 150 & 40 & & $45^{*}$ & 0 & & 0 & 0 & \\
\hline & $\begin{array}{c}\text { Severe congestion and } \\
\text { chemosis }\end{array}$ & 50 & 10 & & $5^{*}$ & 0 & & 0 & 0 & \\
\hline & $\begin{array}{l}\text { Very Severe congestion } \\
\text { and chemosis }\end{array}$ & 50 & 0 & & 0 & 0 & & 0 & 0 & \\
\hline \multirow{4}{*}{ Ocular discomfort } & Mild & 225 & 300 & \multirow{3}{*}{$<0.05$} & $90^{*}$ & 0 & $<0.005$ & 0 & 0 & \multirow{4}{*}{$<0.05$} \\
\hline & Moderate & 50 & 15 & & $30^{*}$ & 0 & $<0.005$ & 0 & 0 & \\
\hline & Severe & 50 & 10 & & $5^{*}$ & 0 & & 0 & 0 & \\
\hline & No & 0 & 0 & & 200 & 325 & & 200 & 450 & \\
\hline
\end{tabular}




\begin{tabular}{|c|c|c|c|c|c|c|c|c|c|c|}
\hline \multirow[t]{4}{*}{ Pain/Photophobia } & No & 200 & 300 & & 250 & 325 & & 250 & 400 & $<0.05$ \\
\hline & Mild & 25 & 25 & & $75^{*}$ & 0 & $<0.005$ & 0 & 0 & \\
\hline & Moderate & 100 & 0 & $<0.005$ & 0 & 0 & & 0 & 0 & \\
\hline & Severe & 0 & 0 & & 0 & 0 & \multirow[t]{4}{*}{$<0.005$} & 0 & 0 & \\
\hline \multirow[t]{4}{*}{ Corneal Status } & Normal & 300 & 275 & \multirow{4}{*}{$<0.05$} & 150 & 325 & & 150 & 500 & $<0.001$ \\
\hline & Keratitis Grade I & 25 & 150 & & $50^{*}$ & 0 & & 0 & 0 & \\
\hline & Keratitis II & 0 & 0 & & $125^{*}$ & 0 & & 0 & 0 & \\
\hline & Keratitis III & 0 & 0 & & 0 & 0 & & 0 & 0 & \\
\hline \multirow[t]{2}{*}{ Lymphadenitits } & Present & 25 & 50 & & 0 & 0 & & 0 & 0 & \\
\hline & Absent & 300 & 275 & & 325 & 325 & & 325 & 325 & \\
\hline \multirow{2}{*}{$\begin{array}{l}\text { Lid edema/Pseudo- } \\
\text { membrane }\end{array}$} & Present & 125 & 145 & & $75^{*}$ & 0 & $<0.003$ & 0 & 0 & \\
\hline & Absent & 200 & 180 & & 250 & 325 & & 250 & 400 & $<0.05$ \\
\hline \multirow[t]{2}{*}{ Intraocular Pressure } & $\begin{array}{c}\text { Normal }(10-21 \mathrm{~mm} \text { of } \\
\mathrm{Hg})\end{array}$ & 325 & 325 & & 325 & 325 & & 325 & 325 & \\
\hline & Raised (>21 mm of $\mathrm{Hg}$ ) & 0 & 0 & & 0 & 0 & & 0 & 0 & \\
\hline \multirow{2}{*}{$\begin{array}{l}\text { Pharyngoconjunctival } \\
\text { Fever }\end{array}$} & Present & 25 & 50 & & 0 & 0 & & 0 & 0 & \\
\hline & Absent & 300 & 275 & & 325 & 325 & & 325 & 325 & \\
\hline \multirow[t]{2}{*}{ Serous discharge } & Present & 150 & 125 & & 0 & 0 & & 0 & 0 & \\
\hline & Absent & 175 & 200 & & 325 & 325 & & 325 & 325 & \\
\hline
\end{tabular}

Table 2: showing the response of treatment in arm one (Conventional) and Intervention arm (arm two). Total number of study subjects $n=650$, total number of eyes treated (in all subjects both eyes were treated) $N=1300$.

* Number of subjects shifted to intervention arm after third week follow up. P value: $<0.05$ is significant for the study and given only where it is relevant.

for the first time had normal visual acuity in both the eyes. These above findings of the study are comparable to the existing knowledge about acute viral conjunctivitis [19]. A slight male predilection in the study may be attributed due to mobility of male subjects and their occupation.

The mean age of study subjects was 27 years of age (range: 6 70 years) in the current study which again, correlated strongly with a study done elsewhere, which concluded that adeno viral acute conjunctivitis is commonest in working age group ranging from 20 - 40 years [20]. Though, again no conclusions can be withdrawn firmly regarding the mean age group in the present study as study subjects ranged from 6 years of age to 70 years and were diverse.

In the present study, in third week follow up, it was conclusive with P value being $<0.05$ that, the patients in arm two had an excellent response to the treatment provided, where as in arm one there were subjects who were taking longer recovery time, developing corneal complications, having visual impairment due to viral keratitis, recurrences, conjunctival pseudo membrane. The study then enrolled subjects from arm one conventional treatment arm to arm two (intervention group) and again these subjects were followed up till 5th week (Table 2). The study though differed from conventional belief with regards to management of acute viral conjunctivitis $[21,22]$. The decision was taken purely based on presentation of the cases in epidemics and the idea was to treat the subject aggressively so that the recovery period is shortened added up by some past researches done in animal models besides interim analysis of the treatment response [23].

Complications occurring due to treatment was negligible in the intervention group which was arm two compared to arm one the conventional group, arm one. However, it is safe to say that the complications which were seen in treatment arm one were due to natural course of the disease and not due to the treatment options. These findings of the study correlate well with the existing literature, that adenovirus of certain species and patients presenting aggressively at first presentation with pseudomembrane respond well to antivirals and topical steroids at initial presentation. When these are not tackled aggressively, they tend to naturally develop various grades of viral keratitis and visual impairment or epithelial infiltrates in the cornea. Although, various researches done in the 
past have varied opinion for and against use of topical steroids in treating acute viral conjunctivitis, these findings were comparable to our study as well as different from our study at the same time $[5,24]$.

The study did not have any subjects with ocular complications at the end of treatment and follow ups, this cannot be entirely attributed to the role played by anti-viral and steroids topically used for the subjects in the treatment, it is without any shade of doubt that excellent response was helped by the natural self-limiting course of the disease as well [1-4]. Another excellent finding in the study was, there was no drop outs of subjects from study due to losing follow up in the first week, this may have been attributed by an excellent counselling done to the study subjects at the initial presentation with regards to the consequences and natural progression of the disease. Which also was added by the fact that minimum time period taken to naturally regress acute viral conjunctivitis varies and is about 10 - 14 days, which made sure subjects came for follow ups as advised [1,6,8,11].

\section{Strengths of the study}

1. This is the first of its kind study where topical steroid and antiviral drugs were used as first line treatment in treating clinically proven acute viral conjunctivitis due to adeno virus in Nepal.

2. The study also forms a treatment protocol for viral keratoconjunctivitis subjects who are refractory to placebo/conventional treatment.

3. This study forms a base to do a larger scale placebo-controlled trial study in other parts of Nepal and also becomes a pilot study for the same.

4. Sample size in the study was big and the duration of the follow up was satisfactory.

\section{Limitations of the study}

1. It was a single blinded study; a double blinded study would further explore to establish the role of antivirals and topical steroids firmly.

2. The study population did not represent pan Nepal population, it was confined to plain western belts of Nepal

3. The findings of the study strongly suggested role of topical steroids along with anti-viral topically to be very positive, however there is no denying of the fact that these drugs should be used judicially and under direct supervision of an Ophthalmologist, misuse of these drugs can lead to devastating complications in the eye.

\section{Conclusion}

Acute viral conjunctivitis is often presented as epidemics and is the commonest cause of infectious conjunctivitis. More than $65 \%$ of acute viral conjunctivitis is caused by adenovirus. No definitive treatment is approved till date for the treatment of acute viral conjunctivitis. The role of topical steroids and anti-viral agents has been controversial in various randomized trials done. Placebo/ conventional treatment are the gold standard treatment even now as the disease process is self-limiting. Certain species of adenovirus are notorious when the cases present with conjunctivitis with Pharyngoconjunctival fever, corneal involvement invariable occur. The study could conclude that if aggressive pulse steroid therapies combined with antivirals are initiated in presumed/diagnosed cases of acute viral conjunctivitis, response to the treatment is excellent in subsequent weeks and days to come. The study also concluded that the subjects receiving the conventional treatment took longer time for recovery, developed visual impairment due to corneal complications of viral infection, and developed the infection in the fellow eye too when unilateral infection was diagnosed.

The study also concluded that the beneficial effects of topical steroids combined with antiviral drugs with antibiotics and soothing agents in eyes with acute viral conjunctivitis are far more superior than the conventional treatment, but steroids should be used with caution under direct supervision of an Ophthalmologist is not advocated for routine red eye cases.

\section{Acknowledgements}

I/We would like to acknowledge the entire subjects attending to the outpatient department of Nepalgunj Medical College with red eyes due to acute viral conjunctivitis and being part of this single blinded study. I would also like to pass my debt to Mrs. Sheela Koirala Bastola for maintaining the data and records of the patients on day to day basis for about two and half years of study. I would like to acknowledge Mr. Som Sharma (Ophthalmic Assistant) for careful counseling of the patients regarding treatment protocol, dosage and follow up time periods.

\section{Conflict of Interest}

None.

\section{Funding}

None. 


\section{Bibliography}

1. Hørven I. "Acute conjunctivitis: a comparison of fusidic acid viscous eye drops and chloramphenicol". Acta Ophthalmologica 71.2 (1993):165-168.

2. Stenson S., et al. "Laboratory studies in acute conjunctivitis". Archives of Ophthalmology 100.8 (1982): 1275-1277.

3. Rönnerstam R., et al. "Prevalence of chlamydial eye infection in patients attending an eye clinic, a VD clinic, and in healthy persons". British Journal of Ophthalmology 69.5 (1985): 385-388.

4. Harding SP., et al. "Adult follicular conjunctivitis and neonatal ophthalmia in a Liverpool eye hospital, 1980-1984". Eye 1.4 (1987): 512-521.

5. Uchio E., et al. "Clinical and epidemiological features of acute follicular conjunctivitis with special reference to that caused by herpes simplex virus type 1". British Journal of Ophthalmology 84.9 (2000): 968-972.

6. Woodland RM., et al. "Causes of conjunctivitis and keratoconjunctivitis in Karachi, Pakistan". Transactions of the Royal Society of Tropical Medicine and Hygiene 86.3 (1992): 317-320.

7. Fitch CP., et al. "Epidemiology and diagnosis of acute conjunctivitis at an inner-city hospital". Ophthalmology 96.8 (1989):1215-1220.

8. Ford E., et al. "Epidemiology of epidemic keratoconjunctivitis". Epidemiologic Reviews 9 (1987): 244-261.

9. Hillenkamp J., et al. "The effects of cidofovir $1 \%$ with and without cyclosporin A 1\% as a topical treatment of acute adenoviral keratoconjunctivitis: A controlled clinical pilot study". Ophthalmology 109.5 (2002): 845-850.

10. Romanowski EG and Gordon YJ. "Efficacy of topical cidofovir on multiple adenoviral serotypes in the New Zealand rabbit ocular model". Investigative Ophthalmology and Visual Science 41.2 (2000): 460-463.

11. Alvaregna L., et al. "Viral Conjunctivitis" In: Krachmer JH, Mannis MJ, Holland EJ. (eds.) Cornea (Vol.1): Fundamentals, Diagnosis, Management. 2nd Edition, Elsevier, Philadelphia, (2005): 629-639.

12. Ishii K., et al. "Comparative studies on aetiology and epidemiology of viral conjunctivitis in three countries of East Asia-Japan, Taiwan and South Korea". International Journal of Epidemiology 16.1 (1987): 98-103.
13. D’Angelo LJ., et al. "Epidemic keratoconjunctivitis caused by adenovirus type 8: epidemiologic and laboratory aspects of a large outbreak". American Journal of Epidemiology 113.1 (1981): 44-49.

14. Heggie AD. "Incidence and etiology of conjunctivitis in Navy recruits”. Military Medicine 155.1 (1990): 1-3.

15. Schrauder A., et al. "Epidemic conjunctivitis in Germany, 2004". Eurosurveillance 11.7 (2006): 7-8.

16. Aoki K and Tagawa Y. "A twenty-one-year surveillance of adenoviral conjunctivitis in Sapporo, Japan". International Ophthalmology Clinics Winter 42.1 (2002): 49-54.

17. Teuchner B., et al. "Tolerability and efficacy of N-Chlorotaurine in epidemic keratoconjunctivitis-a double-blind, randomized, phase-2 clinical trial". Journal of Ocular Pharmacology and Therapeutics 21 (2005): 157-164.

18. Gordon YJ., et al. "The effects of topical nonsteroidal anti-inflammatory drugs on adenoviral replication". Archives of Ophthalmology 116.7 (1998): 900-905.

19. Ishiko H., et al. "Novel human adenovirus causing nosocomial epidemic keratoconjunctivitis". Journal of Clinical Microbiology 46.6 (2008): 2002-2008.

20. Kuo SC., et al. "Corneal superinfection in acute viral conjunctivitis in young children". Journal of Pediatric Ophthalmology and Strabismus 45.6 (2008): 374-376.

21. Wilkins MR., et al. "Randomized placebo-controlled trial of topical steroid in presumed viral conjunctivitis". British Journal of Ophthalmology 95.9 (2011): 1299-1303.

22. Kaufman HE. "Adenovirus advances: new diagnostic and therapeutic options". Current Opinion in Ophthalmology 22.4 (2011): 290-293.

23. Clement C., et al. "Clinical and antiviral efficacy of an ophthalmic formulation of dexamethasone povidone-iodine in a rabbit model of adenoviral keratoconjunctivitis". Investigative Ophthalmology and Visual Science 52.1 (2011): 339-344.

24. Trauzettel-Klosinski S., et al. "The effects of topical steroids in epidemic kerato-conjunctivitis". Klin Monbl Augenheilkd 176.6 (1980): 899-906.

25. Ward JB., et al. "A prospective, double-masked trial of trifluridine, dexamethasone, and artificial tears for treatment of viral conjunctivitis". Ophthalmology 107 (2000): 1512-1517. 
26. Laibson PR., et al. "Corneal infiltrates in epidemic keratoconjunctivitis. Response to double-blind corticosteroid therapy". Archives of Ophthalmology 84.1 (1970): 36-40.

27. Romanoski EG., et al. "Short-term treatment with a potent topical corticosteroid of an acute ocular adenoviral infection in the New Zealand white rabbit". Cornea 20.6 (2001): 657-660.

Volume 2 Issue 9 October 2019

(c) All rights are reserved by Bastola P., et al. 